\title{
How Yellow Is Your Banana? Toddlers' Language-Mediated Visual Search in Referent-Present Tasks
}

\author{
Nivedita Mani \\ University of Göttingen
}

\author{
Elizabeth Johnson \\ University of Toronto
}

\author{
James M. McQueen and Falk Huettig \\ Max Planck Institute for Psycholinguistics, Nijmegen, the Netherlands, and Radboud University Nijmegen
}

\begin{abstract}
What is the relative salience of different aspects of word meaning in the developing lexicon? The current study examines the time-course of retrieval of semantic and color knowledge associated with words during toddler word recognition: At what point do toddlers orient toward an image of a yellow cup upon hearing color-matching words such as "banana" (typically yellow) relative to unrelated words (e.g., "house")? Do children orient faster to semantic matching images relative to color matching images, for example, orient faster to an image of a cookie relative to a yellow cup upon hearing the word "banana"? The results strongly suggest a prioritization of semantic information over color information in children's word-referent mappings. This indicates that even for natural objects (e.g., food, animals that are more likely to have a prototypical color), semantic knowledge is a more salient aspect of toddler's word meaning than color knowledge. For 24-month-old Dutch toddlers, bananas are thus more edible than they are yellow.
\end{abstract}

Keywords: color, semantics, toddlers, word recognition, object recognition

As the developing child learns new words, he or she must of course learn not only the words themselves (the labels) but also what those words mean. A label such as "banana," for example, must come to be associated with semantic knowledge-for instance, that it refers to something that can be eaten-and with color-based knowledge - that bananas are typically yellow. The current study examines whether toddlers retrieve and use both types of knowledge during word-referent mapping and pay attention to the semantic properties as well as to visually salient perceptual attributes such as the color knowledge associated with words and objects. Furthermore, we ask whether there are differ-

This article was published Online First July 30, 2012.

Nivedita Mani, Language Acquisition Junior Research Group, University of Göttingen, Göttingen, Germany; Elizabeth Johnson, University of Toronto, Toronto, Ontario, Canada; James M. McQueen, Max Planck Institute for Psycholinguistics, Nijmegen, the Netherlands, and Donders Institute for Brain, Cognition, and Behaviour, and Behavioural Science Institute, Radboud University Nijmegen, Nijmegan, the Netherlands; Falk Huettig, Max Planck Institute for Psycholinguistics, Nijmegen, the Netherlands, and Donders Institute for Brain, Cognition, and Behaviour, Radboud University Nijmegen.

Support was provided by the German Initiative of Excellence (Institutional Strategy) to Georg-August-Universität Göttingen. Additional support was provided by the Max Planck Society and an NWO Spinoza Prize entitled "Native and Non-Native Listening" awarded to Anne Cutler. We thank Angela Khadar and the rest of the Nijmegen Baby Lab crew, as well as all of the participating families, for their assistance with this study.

Correspondence concerning this article should be addressed to Nivedita Mani, Language Acquisition Junior Research Group, University of Göttingen, Gosslerstrasse 14, 37073 Göttingen, Germany. E-mail: nmani@gwdg.de ences in the time-course of children's retrieval of semantic and color knowledge. Do children orient faster to semantic matching images relative to color matching images, for example, orient faster to an image of an apple relative to a yellow shirt, upon hearing the word "banana"?

By 4 months of age, infants display an adultlike ability to discriminate between basic color categories (Bornstein, Kessen, \& Weiskopf, 1976; Catherwood, Crassini, \& Freiberg, 1989; Franklin \& Davies, 2004; Hayne, Rovee-Collier, \& Perris, 1987). However, despite this precocious sensitivity to color categories, the color of an object appears to be a nonsalient cue in guiding infant object categorization or object individuation (Wilcox, 1999; Wilcox, Woods, Chapa, \& McCurry, 2007): It is only around 11.5 months of age that infants use color as a basis for individuating objects, for example, individuating a red ball and a green ball (Wilcox et al., 2007). This reduced salience of color is typically attributed to the importance of color to the individuation or categorization of objects - the color of most objects is arbitrary, with little relevance to their category membership or their identity.

Given the reduced salience of color in infant object categorization, it would be reasonable to expect the color of objects to be a nonsalient characteristic of the labels of these objects-if objectcategory representations are underspecified with respect to color (as indicated by object individuation tasks), the labels referring to these categories should be equally underspecified for color information. That is, in contrast to the speed and accuracy with which toddlers retrieve shape-based, semantic, and phonological representations of words (Arias-Trejo \& Plunkett, 2009; Landau et al., 1988; Mani \& Plunkett, 2010), color representations of words may not be as readily available in toddler speech processing. 
Indeed, the robustness of an influence of color knowledge on toddler word recognition is debatable. On the one hand, children do not extend novel labels to color matching/shape mismatching objects but do overextend novel labels to color mismatching/shape matching objects (Graham \& Poulin-Dubois, 1999). This suggests that the novel labels are underspecified with respect to color to the extent that the same novel label can more easily be associated with two color representations than with two shape representations. In contrast, words are associated with highly specific phonological, shape, and semantic representations-at least till around 18 months of age when words may refer to atypical category members (Meints, Plunkett, \& Harris, 1999) or till 3-years of age, when children first extend words at the superordinate level (Liu, Golinkoff, \& Sak, 2001).

On the other hand, recent research (Johnson \& Huettig, 2011) shows that color knowledge retrieved on hearing familiar words can drive toddler fixations to color matching competitors in visual search tasks (see Huettig \& Altmann, 2011, for corresponding effects in adults). In this work, 3-year-olds were presented with images of two objects on a screen and asked to find an object not present on the screen (henceforth the prime label). For example, children were presented with a picture of a red and green ball and asked to find the frog. Crucially, in some trials, the color of the object associated with the prime word (i.e., "frog"), matched the color of one of the objects on the screen (i.e., the green ball). Approximately $1 \mathrm{~s}$ after the onset of the prime, infants showed a significant preference to fixate the color-matching object (i.e., the green ball) relative to the color-mismatching object. That is, children looked more toward the color-matching object in trials where the referent of prime label overlapped in color with the target image (color-matching trials) relative to trials where the prime and target did not overlap in color (unrelated trials). Johnson and Huettig's (2011) results, therefore, suggest that children readily retrieve color representations of spoken words and that color knowledge modulates shifts in toddler visual attention.

However, Johnson, McQueen, and Huettig (2011) failed to find a reliable difference in the time-course of retrieval of semantic and color knowledge in 24-month-old word processing. Johnson et al. replicated color-mediated shifts in visual attention in 2-year-olds and showed that this behavior is also observed in tasks where the images on screen differ in more than the color dimension: Children were presented with pairs of images depicting different objects (e.g., a blue chair and a red cup) and asked to find an object not present on screen (e.g., a strawberry). Once again, $1 \mathrm{~s}$ after the onset of the prime word (strawberry), toddlers fixated the image of the red cup more than blue chair, providing stronger evidence for the retrieval of knowledge about color representations in word recognition. Importantly, the 2-year-olds in Johnson et al. (2011) were also presented with semantic-matching trials, that is, when the prime word (strawberry) was semantically related to one of the objects on screen. As with color-matching trials, $1 \mathrm{~s}$ after naming, children looked longer toward the property-matching objects in semantic-matching trials relative to unrelated trials. While the 24-month-olds displayed a trend toward differences in the timecourse of retrieval of semantic and color knowledge, this difference was not significant, suggesting that orientations toward semantic-matching objects as well as color-matching objects took place only $1 \mathrm{~s}$ after naming.
Furthermore, we note that the time-course of semantic priming effects in Johnson et al. (2011-significant effects of semantic relatedness only in the time-period of 1-2 s after naming) contrasts with other studies reporting differences between semantically related and unrelated trials aggregated over the entire postnaming window (233 ms to $2,000 \mathrm{~ms}$ from the onset of the target label, Arias-Trejo \& Plunkett, 2009; Styles \& Plunkett, 2011). In both studies reported above (Johnson \& Huettig, 2011; Johnson et al., 2011) children were asked to find an object not present on the screen (referent-absent task), that is, children were presented with a picture of some cookies and asked to find a strawberry. In contrast, studies reporting earlier and long-lasting effects of semantic priming (Arias-Trejo \& Plunkett, 2009; Styles \& Plunkett, 2011) employed a more prototypical priming task, where children were presented with both a prime and a target label (referentpresent task). That is, children were presented with a picture of a dog and a boat and heard the carrier phrase "I saw a cat ___ dog" with a $200 \mathrm{~ms}$ interstimulus interval between prime (cat) and target label (dog).

Might the fact that Arias-Trejo and Plunkett (2009) and Styles and Plunkett (2011) present children with a referent-present task as opposed to a referent-absent task (Johnson et al., 2011) explain the differences in the time-course of retrieval of semantic knowledge across the studies? Perhaps the delay in children orienting toward semantic or color-matching objects in Johnson et al. (2011) is indicative of the cognitive load involved in comprehending absent reference (Saylor, 2004), that is, indicative of the additional demands of finding a match between the mismatching command (Where's the frog?) and one of the pictured images (i.e., a green ball). Indeed, evidence for delays in referent-absent comprehension is common, with children taking longer to respond to the name of referent-absent objects (Ganea, 2005). That is, children may retrieve semantic and color knowledge (e.g., that frogs are green) immediately upon hearing the mismatching label but may be delayed in orienting to the green object due to their search for a matching image. Taken together, there appears to be considerable variability not just with regard to the time-course of the effects reported by Johnson et al. (2011); Arias-Trejo and Plunkett (2009) and Styles and Plunkett (2011) but also with regard to the finding of an effect at all-Styles and Plunkett (2011) report not finding a semantic priming effect in referent-absent tasks in contrast to Johnson et al. (2011).

Given that referent-present tasks appear to be able to tap into earlier and longer-lasting effects of priming, the current study seeks to reexamine the time-course of retrieval of color and semantic knowledge in toddler word processing using a referentpresent task. Do children show retrieval of both semantic and color knowledge earlier than $1 \mathrm{~s}$ after naming, in contrast to what was demonstrated by Johnson et al. (2011)? Importantly, might we be able to tap into differences in the time-course of retrieval of semantic and color knowledge, as suggested by Johnson et al. (2011)?

We employ a primed intermodal preferential looking task to examine the time-course of retrieval of color and semantic information in referent-present tasks (see Arias-Trejo \& Plunkett, 2009; Styles \& Plunkett, 2011). We used the same stimulus pairs as in Johnson et al. (2011) to allow comparability of results across the two studies, that is, the current study and Johnson et al. The main difference between the current study and Johnson et al. (2011) is 
that, in the current study, after being presented with the prime sentence, toddlers also heard the label for one of the images on the screen (the target label). That is, we presented children with semantic-matching trials (e.g., children heard "I saw a banana cookie" in the presence of an image of a cookie), colormatching trials (e.g., children heard "I saw a banana __ cup" in the presence of an image of a yellow cup) and unrelated trials (e.g., children heard "I saw a table __ cup" in the presence of an image of a yellow cup). In addition, we also presented children with neutral trials (e.g., children heard "I saw somethingcookie" in the presence of an images of a cookie). For ease of understanding, Figure 1 presents a schematic of trials in the current study, in Johnson et al. (2011) and in Arias-Trejo and Plunkett (2009). We compare color- and semantic-based effects in the referent-present situation and examine the relative salience of these two types of knowledge.

Thus, across trials, children were presented with the label for the target image primed by a color-matching prime, a semanticmatching prime, an unrelated prime, or a neutral prime. If children retrieve color-knowledge, we expect them to orient toward the target image more in color-matching trials with respect to unrelated or neutral trials. Similarly, if children retrieve semantic knowledge in word processing, we expect them to orient toward the target image more in semantic-matching trials relative to unrelated or neutral trials. Finally, if, as with adults, conceptual knowledge is indeed more salient than color knowledge (cf. Huettig \& Altmann, 2011), we expect children to orient toward the target image earlier in semantic-matching trials (e.g., cookie, given the prime "banana"), compared to color-matching trials (e.g., the yellow cup, given the prime "banana").

\section{Method}

\section{Participants}

A total of 45 Dutch-learning children at 2 years of age were tested $(M=754$ days, range $=743-760$ days; $S D=4.49,21$ male). Six children were excluded from analysis due to their not completing the experiment (2), due to problems during calibration (1) or due to their not providing reliable eye-tracking data for at least two trials per condition (3). Children came from a sample of mostly middle-class families who responded to an invitation letter sent to all families living with infants of appropriate age in the Nijmegen, Netherlands, area. All children came from families where Dutch was the only language spoken at home. The children were healthy, full-term infants without any pre- or perinatal complications. Parents gave informed consent for participation of their child in the study.

\section{Stimuli}

Twelve Dutch words served as critical prime labels for the color and semantic matching trials. They were characterized by a typical color and by belonging to a distinct semantic category (e.g., banaan, banana). These 12 words were paired with targets that matched in either color (e.g., a yellow cup) or semantic category (e.g., cookie). Prime sentences were always recorded in the carrier phrase $I k z a g$, "I saw." Target words were spliced into the prime sentence $200 \mathrm{~ms}$ after the offset of the prime word. Thus, for example, stimuli for a color-matching trial included the sentence "I saw a banana __ cup" and images of a yellow cup and a blue chair. Stimuli for semantic-matching trials included the sentence "I saw a banana ___ cookie" and images of a cookie and a bicycle. In addition to critical color or semantic-matching prime-target sentences, we also recorded unrelated prime-target sentences, where the targets were paired with one of four unrelated prime labels (i.e., soap, house, telephone, and table), for example, "I saw a house _ cup." Finally, we recorded neutral prime-target sentences, where targets were primed by a neutral sentence: $I k$ zag iets, "I saw something cup" (see Table 1 for a list of stimuli).

Aside from the neutral prime sentences which were novel to the current study, the pairing of primes and targets maintained the pairing of visual (not labeled) and verbal targets (not imaged) in
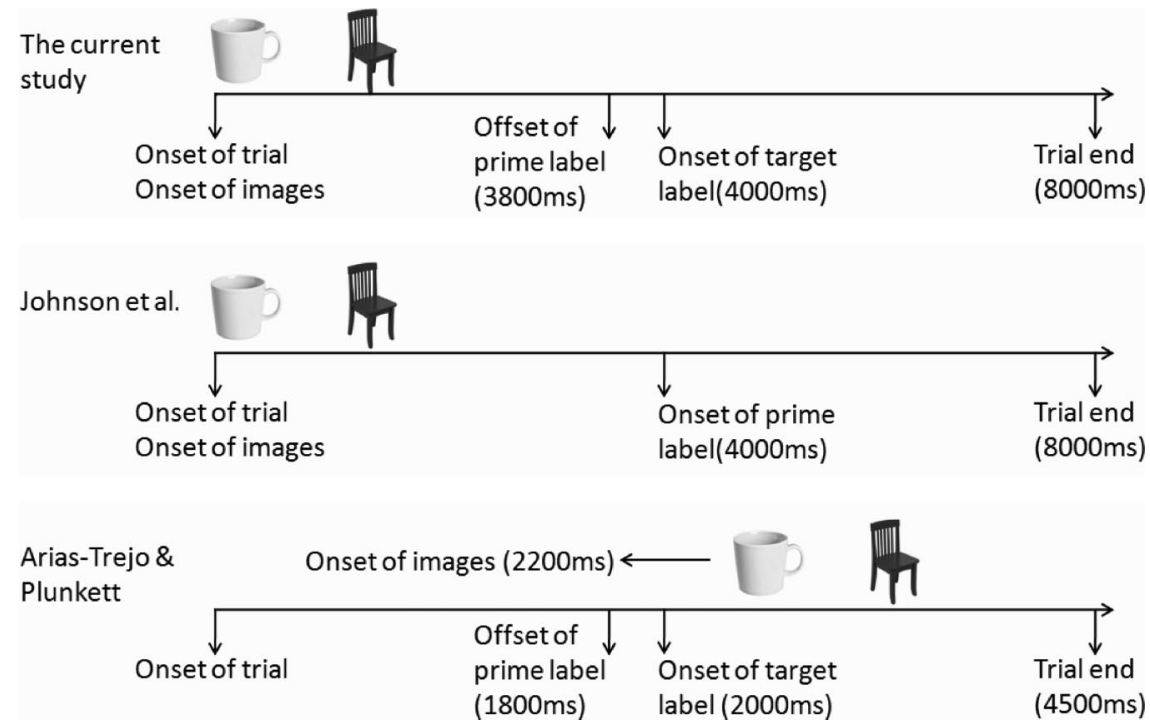

Figure 1. Schematic of trials in the current study, in Johnson et al. (2011), and in Arias-Trejo and Plunkett (2009). 
Table 1

Pairing of Prime-Target Items (Translated From Dutch)

\begin{tabular}{|c|c|c|c|c|c|}
\hline \multirow[b]{2}{*}{ Carrier sentence } & \multicolumn{2}{|c|}{ Related prime } & \multirow[b]{2}{*}{ Unrelated prime } & \multirow[b]{2}{*}{ Neutral prime } & \multirow[b]{2}{*}{ Target } \\
\hline & Prime & Type & & & \\
\hline I saw & a deer & Color & soap & something & brown bag \\
\hline I saw & a monkey & Color & a house & something & brown mitten \\
\hline I saw & chocolate & Color & soap & something & brown sock \\
\hline I saw & a crocodile & Color & a telephone & something & green sock \\
\hline I saw & a frog & Color & soap & something & green truck \\
\hline I saw & an elephant & Color & a table & something & grey truck \\
\hline I saw & an orange & Color & a house & something & orange couch \\
\hline I saw & a pig & Color & a table & something & pink bag \\
\hline I saw & a strawberry & Color & soap & something & red cup \\
\hline I saw & a tomato & Color & soap & something & red mitten \\
\hline I saw & fries & Color & a telephone & something & yellow couch \\
\hline I saw & a banana & Color & a table & something & yellow cup \\
\hline I saw & a deer & Semantic & a table & something & turtle \\
\hline I saw & a monkey & Semantic & a table & something & $\operatorname{dog}$ \\
\hline I saw & chocolate & Semantic & a house & something & apple \\
\hline I saw & a crocodile & Semantic & a table & something & $\operatorname{dog}$ \\
\hline I saw & a frog & Semantic & a telephone & something & bird \\
\hline I saw & an elephant & Semantic & a telephone & something & fish \\
\hline I saw & an orange & Semantic & a house & something & sandwich \\
\hline I saw & a pig & Semantic & soap & something & turtle \\
\hline I saw & a strawberry & Semantic & a house & something & cookies \\
\hline I saw & a tomato & Semantic & a telephone & something & bread \\
\hline I saw & fries & Semantic & a telephone & something & grapes \\
\hline I saw & a banana & Semantic & a house & something & cookies \\
\hline
\end{tabular}

Johnson et al. (2011). The difference across studies was that the visual targets were also labeled in the current study. Thus, while primes were always only auditory (as in Johnson et al., 2011), targets were presented both auditorally and visually (unlike the visual-only presentation in Johnson et al., 2011).

Visual stimuli were computer images created from photographs, with one image for each target word. Target images were paired with distractor images that did not overlap with the prime or the target in semantic, phonological, shape, or color properties. Auditory and visual stimuli for the test trials were combined together to make short movies of $8 \mathrm{~s}$ duration for each trial. Movies were formatted such that the target and distractor images (measuring $480 \times 380$ pixels each) appeared simultaneously to the left and right of the screen $(1280 \times 1024$ pixels $)$ with a separation of 320 pixels between them.

\section{Experimental Set-Up}

During the experiment, gaze data from both eyes was recorded using a Tobii 1750 eye tracker. The Tobii eye-tracking system is integrated into a 17-inch TFT flat-screen monitor on which the stimuli were presented. The eye-tracker records gaze data at $50 \mathrm{~Hz}$ with an average accuracy of $0.5^{\circ}$ visual angle. The monitor was mounted on an adjustable arm, so that the screen could always be positioned approximately $60 \mathrm{~cm}$ away from the child's face. Children sat on their caregiver's lap throughout the procedure. Prior to testing, we calibrated the gaze of each child using a 9-point calibration procedure, in which an attention-getter appeared in every position of a $3 \times 3$ grid of calibration points. The experiment started if eight or more points were successfully calibrated.

\section{Procedure}

The short movies were combined to form six different lists of trials. Children were randomly assigned to one of these lists. Each list included 12 test trials. Of the 12 test trials, four were unrelated trials (i.e., where the prime and target were unrelated to one another), four were neutral trials (i.e., where the targets were primed by the neutral prime sentence "I saw something"), and four were related trials (i.e., where the prime and target were either color matching [2 trials] or semantic matching [2 trials]). In addition, we included attention-getter trials $2 \mathrm{~s}$ in duration every second trial where children were presented with a purple Teletubby moving up and down the screen in tune to a chime. The attention-getter trials maintained children's attention to the screen during the experiment.

The 12 test trials presented children with a target and a distractor image and the label for the target image primed by the related/ unrelated/neutral prime sentence (i.e., Ik zag een [Prime] ... [Target], "I saw a [Prime] ... [Target]"). Each test trial began with two images (i.e., a target and a distractor image) presented simultaneously onscreen in silence. Target and distractor images appeared either to the left or to the right of the screen as in previous studies (Johnson et al., 2011; Arias-Trejo \& Plunkett, 2009,- see section on stimuli for an exact description of the visual stimuli). The images remained on screen for the entire $8 \mathrm{~s}$ duration of the trial, that is, all trials had a fixed duration of $8 \mathrm{~s}$. A variable duration after the onset of the trial, the carrier sentence began such that the offset of the prime word was at 3,800 ms. The target label began $200 \mathrm{~ms}$ after the offset of the prime label (i.e., 4,000 ms into the trial; see Figure 1 for a schematic of these trials). 
The only relation between targets and primes in related trials was the shared color or shared semantic attribute (food, animal). There was no relation between the primes and targets in unrelated trials. In neutral trials, the prime was iets, "something," followed by a target label. The distractor image was never labeled, and the label for the distractor image was unrelated to the target or prime label. The list of prime and target stimuli is given in Table 1. Children saw each image only once during the experiment. Targetdistractor pairs appeared in all conditions with equal frequency across children. Targets appeared equally often to the left and to the right in all conditions. Order of presentation of trials was pseudorandomized for each list, with an attention-getter trial between every two test trials.

The experiment took $1 \mathrm{~min} 46 \mathrm{~s}$ to complete. During the experiment, parents were asked to close their eyes and not direct children's attention to any part of the screen.

\section{Analysis}

Areas of interest were defined according to the size of the target and distractor images, that is, $480 \times 380$ pixels each. There was no spatial overlap between the Areas of interest for target and distractor (separated by 320 pixels). The eye-tracker provides an estimate of whether children were looking at the target or distractor image for each time-stamp during the trial, with one data-point every $20 \mathrm{~ms}$. Data from time-stamps were only included when the eye-tracker reported validity less than or equal to 2 on the Tobii validity scale (which indicates that the eye-tracker has reliably acquired data from one or both eyes of the participant). Gaze data from the eye-tracker was aggregated into $40 \mathrm{~ms}$ bins such that each $40 \mathrm{~ms}$ bin (across the duration of the trial) was coded for whether infants were looking at the target, the distractor or elsewhere. Since binning produces aggregated data, bins were marked as looking toward the target or the distractor only if children looked at the same image in both time-stamps within a bin. Bins where children did not look at the same image in both time-stamps were marked as looking elsewhere (i.e., neither at the target not at the distractor). This allowed us to calculate for each $40 \mathrm{~ms}$ bin whether the child was looking at the target, the distractor, or elsewhere. These bins were further aggregated across three time windows. The first window, the baseline window, counted all fixations that occurred 1,000 ms before the onset of the prime label. The second window, the prime window, counted all fixations that occurred from the onset of the individual prime labels to the onset of the target label. The third window, the target window, counted all fixations that occurred from the onset of the target label to 1,000 ms posttarget-label-onset. For each window, we determined the amount of time infants looked at the target (T) and distractor (D) images. We then calculated the proportion of time $[\mathrm{T} /(\mathrm{T}+\mathrm{D})]$ infants spent looking at the target in each window. This proportional measure was our dependent variable. Only those trials in which infants fixated both the target and the distractor image in the prenaming phase were included (see Mani \& Plunkett, 2007, 2008, 2010, for other studies employing similar exclusion criteria). This criterion excluded $6 \%$ of trials. We used this exclusion criterion to help eliminate trials where infants were not on task, based on the requirement that participants explore the full visual array before identifying potential matches between image and label.
Prior to their visit to the laboratory, parents were asked to fill out a standardized vocabulary inventory (of 600 words) to indicate whether their children understood and/or produced the items presented to them in the experiment as well as the colors manipulated in the task. This allows an additional analysis where we only consider those trials where children were reported to know the words presented to them. As there were no differences in the pattern of children's responding across the two analyses, the main analyses are reported using overall data. Preliminary analyses also ruled out main effects of participant gender $(p=.32)$ and of the trial lists that participants were randomly allocated to $(p=.78)$, so the data in further analyses were pooled across these variables.

\section{Results}

Figure 2 plots the proportion of children's fixations to the target across the three crucial time-windows. The data plotted in Figure 2 suggest that there was no difference in children's fixations to the target across all conditions in the baseline window (preprimeonset). However, in the first second after the onset of the prime and even before the onset of the target label (the prime window), children showed a greater preference for the target image in semantic-matching trials compared to any other condition. Note that the baseline and prime windows marked in Figure 2 indicate only the average baseline and prime windows. Analysis was based on prime windows set for each trial on the basis of the onset of individual prime labels. In the first second after the onset of the target label (the target window), children showed a greater preference for the target image in color-matching trials compared to any other condition. There was no difference between unrelated trials and neutral trials across all three time-windows $(p s>.5)$. Further statistical analysis, therefore, pooled the data from neutral and unrelated trials (forming a single control condition).

\section{Baseline Window (1,000 MS Prior to Onset of Prime Label)}

A repeated-measures ANOVA with condition as (colormatching, semantic-matching, control) a within-subjects factor found no effect of condition in this time window, $F(2,37)=0.04$, $p=.96, \eta^{2}=.002$. Planned post hoc comparisons also found no significant differences between conditions $(p s>.6)$. Also, children did not look at the target image above chance in any condition $(p s>1)$.

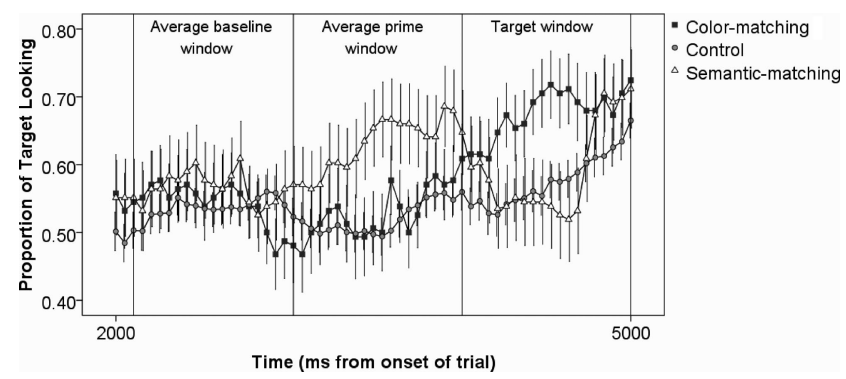

Figure 2. Time-course of children's responding across baseline, prime, and target windows. Error bars indicate $+/-$ one standard error from the mean. 


\section{Prime Window (Onset of Prime Label to Onset of Target Label)}

An analogous repeated-measures ANOVA found a nearsignificant effect of condition in this time window, $F(2,37)=$ $2.91, p=.067, \eta^{2}=.14$. Planned post hoc comparisons showed that children were more likely to fixate the target in semanticmatching trials $(M=.64, S D=.27)$ compared to color-matching trials $(M=.53, S D=.23), t(38)=1.98, p=.05, d=0.32$, or control trials $(M=.53, S D=.16), t(38)=2.09, p=.043, d=$ 0.38 . Note that this difference was not an artifact of pooling the data for neutral and unrelated trials, since similar results were obtained when these trials were separately analyzed. Children were more likely to fixate the target in semantic-matching trials compared to neutral-prime trials $(M=.51, S D=.22), t(38)=2.25$, $p=.03, d=0.38$, or unrelated trials $(M=.54, S D=.17), t(38)=$ $2.09 ; p=.043, d=0.38$. There was no difference between any of the other conditions ( $p s>.5$ ). During this time-window, children looked at the target above chance (0.5) in semantic-matching trials, $t(38)=3.27, p=.002, d=0.51$, and not in any other condition $(p \mathrm{~s}>.2)$.

\section{Target Window (0 to 1,000 MS Postonset of Target Label)}

A further analogous ANOVA found a significant effect of condition in the target time window, $F(2,37)=4.59, p=.017$, $\eta^{2}=.19$. In contrast to the earlier prime window, planned post hoc comparisons showed that children were more likely to fixate the target in color-matching trials $(M=.71, S D=.22)$ compared to control trials $(M=.57, S D=.14), t(38)=2.59, p=.013, d=$ 0.52 . Yet again, this finding was replicated when neutral and unrelated trials were separately analyzed. Children were more likely to fixate the target in color-matching trials compared to neutral-prime trials $(M=.58, S D=.20), t(38)=2.37, p=.023$, $d=0.41$, or unrelated trials $(M=.56, S D=.24), t(38)=2.59$, $p=.013, d=0.44$. There was no difference between any of the other conditions $(p s>.5)$.

There was also a near-significant trend toward a difference between color-matching and semantic-matching trials $(M=.60$, $S D=.31)$ in this time-window, $t(38)=1.8, p=.078, d=0.31$. Given the presentation of the target label in this time-window, children looked at the target above chance in every conditioncolor-matching, $t(38)=5.78, p<.001, d=0.95$, semanticmatching, $t(38)=2.04, p=.048, d=0.32$, and control, $t(38)=$ $3.2, p=.002, d=0.5$.

\section{Vocabulary Analysis}

A separate analysis only included those trials where children were reported to know the words presented to them in the study (based on parental communicative development inventory measures). This criterion excluded $14 \%$ of trials. This analysis yielded a very similar pattern of responding. There was a significant difference between semantic-matching trials and control trials in the prime window, $t(38)=1.97, p=.05, d=0.37$, and a significant difference between color-matching trials and control trials in the target window, $t(33)=2.23, p=.027, d=0.69$. All other comparisons were not significant.

\section{Discussion}

The current study set out to examine the time-course of retrieval of color and semantic knowledge in toddler word processing. We argued that while previous results found no reliable difference in the time-course of retrieval of color and semantic knowledge, reexamination of this issue was required due to differences in the tasks used in Johnson et al. (2011) and other studies investigating semantic priming with toddlers (Arias-Trejo \& Plunkett, 2009; Styles \& Plunkett, 2011). We suggested that, first, the task used in the current study (and Arias-Trejo \& Plunkett, 2009; Styles \& Plunkett, 2011; i.e., the referent-present task) might be able to tap into the time-course of retrieval of color-knowledge in word processing and, second, that such tasks might highlight differences in the time-course of retrieval of semantic and color-knowledge. Examining the retrieval of color and semantic knowledge in toddlers using a range of tasks is required to provide a fuller picture of the processes underlying word recognition in young children, especially in this referent-present situation, where no knowledge about the prime labels had to be retrieved.

With regard to the first question, the results of this study strongly support the conclusion of Johnson et al. (2011) that infants readily retrieve color-knowledge during language-mediated visual search. Despite being presented with the target label, thereby facilitating an easy match between auditory and visual stimuli, children continued to show a greater preference for the color matching object when primed by a color-matching label compared to an unrelated label. That is, when presented with a yellow cup, a blue chair, and the label "cup," children showed a greater preference to look at the yellow cup when primed by a color-matching prime (banana) compared to a neutral prime (something) or an unrelated prime label (house). There were no differences in the time-course of children's retrieval of color knowledge between Johnson et al. (2011) and the current studythis effect appeared $1 \mathrm{~s}$ after the onset of the prime label and persisted even when children were presented with the appropriate target label.

With regard to the second question, there were crucial differences between the results of the current study Johnson et al.'s (2011) findings. These differences hinge on the relative timecourse of retrieval of semantic and color knowledge. Johnson et al. found no reliable differences in the time-course of retrieval of semantic and color knowledge with toddlers orienting toward the matching object only $1 \mathrm{~s}$ after the onset of the semantically or color-related label. In contrast, the current study found effects of semantic relatedness even as children heard and processed the semantically related prime label. Note that inspection of Figure 2 in Johnson et al. (2011) reveals a trend toward such a finding, but this difference was not statistically significant. The current experiment, therefore, builds upon Johnson et al.'s findings to chart the differential time-course of retrieval and/or use of semantic and color information. Semantic overlap resulted in earlier increased overt attention to the target object than color overlap, with children orienting to color-matching objects approximately $1 \mathrm{~s}$ after they oriented to semantic-matching objects.

The differential time-course of children's orientation to semantic-matching and color-matching objects suggests that semantic knowledge, relative to color-knowledge, is particularly central to toddlers' word meaning. Prioritization of semantic (e.g., 
category and functional) knowledge over color knowledge may seem reasonable with regard to manmade objects, which tend to be low in color diagnosticity (chairs naturally vary in color). The case is more interesting for words referring to natural objects, such as the prime words used in the present study. In the case of natural objects, where there is less natural variation in color, it is conceivable that color knowledge (that frogs in Northern Europe tend to be green and strawberries, red) may be as salient to word meaning as categorical and functional knowledge (that frogs are animals and that strawberries can be eaten). Nevertheless, with regard to our prime words, our study suggests that, even for natural objects, semantic knowledge is a particular salient aspect of toddlers' word meaning, more so than color-knowledge.

It is also important to note that the current study finds a significant difference not just between related trials (color-matching, semantic-matching) and unrelated trials but also between related trials and neutral trials (i.e., those trials where the prime was a neutral word-something [iets]—that neither matched nor mismatched with the perceptual and conceptual properties of the target). Indeed, interpretation of the difference between unrelated trials and related trials is complicated-the difference could be driven either by interference with target recognition in unrelated trials (due to mismatch in the perceptual or conceptual properties of the prime and target) or by facilitation in target recognition in related trials (due to perceptual or conceptual overlap between the prime and target label). However, the finding of a facilitatory effect of target recognition in related trials compared to neutral trials clarifies this distinction. The prime in neutral trials was underspecified with respect to its perceptual or conceptual features and could not, therefore, mismatch with the perceptual and conceptual features of the target. A facilitatory effect of target recognition in related trials relative to neutral trials confirms, therefore, that perceptual or conceptual overlap facilitates target recognition, perhaps by earlier retrieval of the overlapping features in related trials.

The results of the current study also provide an interesting contrast with previous studies of semantic priming, in particular with the results of Arias-Trejo and Plunkett (2009). Arias-Trejo and Plunkett (2009) employ a similar semantic priming task and find that children showed a greater preference for looking at the target image when primed by semantically and associatively related labels as opposed to unrelated labels. Our study finds a similar effect with only semantically but, crucially, not associatively related labels by 24 -months of age. That is, despite the absence of associative links between primes and targets in the current experiment, we find strong evidence for the prioritization of semantic over color knowledge. This suggests a particularly important role for semantic knowledge (and not just associative knowledge) about words in toddler word processing. Given the absence of associative links between the words tested in the current experiment, it would be interesting to see whether greater prioritization of semantic over color knowledge is found in tasks where primes and targets are both semantically and associatively related, for example, apple-orange relative to chocolate-orange.

We note, however, that the time-course of effects does differ across the two studies. In Arias-Trejo and Plunkett (2009), children showed a preference for the target in semantically related trials in the time-window following the target label. In the current study, children show increased target looking in semantically related trials (relative to unrelated trials) immediately following the prime label but not in the time-window following the target label (i.e., 0 to $1,000 \mathrm{~ms}$ after target label onset). We suggest that this is due to children already showing a significant preference for the semantically related target in the prime window in the current study - children could not demonstrate such a preference in AriasTrejo and Plunkett (2009) study due to the images not being presented to them concurrently with the prime label. In visual search tasks, once an object has been processed, there is typically some short-term inhibition of renewed attention to the same object (see Grison, Paul, Kessler, \& Tipper, 2005, for a review of objectbased inhibition of return).

We now turn to the contrast between Johnson et al. (2011) and the current study, which we suggest lends itself to discussion of the cues that children might be using to make word-referent mappings. Given that we find effects of semantic-overlap and color-overlap in both referent-present and referent-absent designs, children, by 24-months of age, appear able to retrieve enough information about labels associated with images in order to make a mapping between a heard word and a presented image. That is, in the referent-absent version of the current study, children are able to detect overlap between a label and an image despite not being presented with the label for this image. On the one hand, this might suggest that orientation toward the feature-overlapping image is based on children's generation of the labels for the presented images (Mani \& Plunkett, 2010) and their detection of featureoverlap between the representations associated with these labels. On the other hand, this might equally suggest that orientation toward feature-overlapping images is driven by children's generation of the features associated with the prime label and their detection of the overlap between these retrieved features and the associated images (Swingley \& Fernald, 2002).

While the current results do not allow us to choose one of these explanations over the other, it is noteworthy that we find similar effects of the retrieval of semantic and color-knowledge between a label and a semantic/color-overlapping image (referent-absent task) relative to a label and a semantic-/color-overlapping label (referent-present task). Nevertheless, the prioritization of semantic- over color-knowledge found in the current study relative to Johnson et al. (2011) might suggest more salient links between semantically related words (e.g., the words banana-cookie), relative to semantically related label-object pairings (e.g., the word banana and the image of a cookie), color-related words, and color-related label-object pairings. The greater salience of wordword relationships relative to word-image relationships is supported by Styles and Plunkett's (2011) findings of no effects of taxonomic and associative overlap between words and images (in a referent-absent task) in contrast to effects of semantic priming between words (in a referent-present task). We suggest that the contrast between the Styles and Plunkett (2011) and Johnson et al. (2011) studies is attributable to the dynamics of the tasks presented to the children. Johnson et al. (2011) presented children with a longer prenaming baseline phase (where the images were presented in silence) than Styles and Plunkett (2011). Longer familiarization with the images may enhance the likelihood of observing semantic relatedness effects in referent-absent tasks.

What are the consequences of our findings for our understanding of the development of children's representations of words? We note that the children tested in the current study were the same age 
as the children showing semantic priming effects in Johnson et al., (2011), Arias-Trejo and Plunkett (2009), and Styles and Plunkett (2011). However, Arias-Trejo and Plunkett (2011) found that 21-month-olds show semantic priming effects only with primes that are both taxonomically and associatively related to the targets. Thus, we might not expect a similar prioritization of purely semantic-knowledge over color-knowledge with children younger than those tested in this experiment. Indeed, this is a hypothesis worthy of future investigation - is the prioritization of semantic knowledge contingent on the maturity of children's semantic representations of words at 24 months of age?

This notion is supported by the finding that our results are not without precedence in the adult literature. Using a cross-modal priming task, Moss, McCormick, and Tyler (1997) found that mature language users retrieve semantic aspects of word meaning (aspirin-pain, cf. Huettig \& Altmann, 2005; Yee \& Sedivy, 2006) before perceptual properties about the visual form of a word's referent (aspirin-white). Our findings fit well with the notion that the strength of retrieval of particular representations predicts the probability of attending toward similar (vision-derived) representations (cf. Huettig, Olivers, \& Hartsuiker, 2011). Semantic knowledge (e.g., whether a certain object is edible) is often more important and relevant to toddlers' daily lives than stored knowledge about an object's typical color (even for fruits and vegetables). We suggest that these differences in informational value underlie the time-course differences in toddler's shifts in eye gaze to semantic and color competitors and that these differences may also influence the way in which lexical representations develop. There may thus be prioritization of semantic information over color information in children's word-referent mappings not only at the time scale of the eye fixations within the trials of the present experiment but also at the time scale of the emergence of the lexicon through early childhood.

Recent work with adults shows that color-mediated shifts are driven by perceived surface color rather than stored color attributes associated with words (Huettig \& Altmann, 2011) — that is, adults show little or no effects of color-overlap between spoken words and visually presented objects when the objects are presented in black and white or in atypical colors. It would be interesting to see whether there is a similar reduced attention to color-overlap between words and atypical objects in childhood. Ongoing work is currently testing a variant of this hypothesis by examining whether children show increased color-mediated shifts when the prime and target overlap in conceptual color (e.g., frogs and pears are both conceptually green) relative to when the prime and target overlap in surface color only (e.g., frogs and green chairs overlap only in surface color).

To conclude, when hearing spoken words whose referents are associated with a prototypical color (e.g., "banana"), 24-montholds are more likely to fixate a color-matched distractor (e.g., a yellow cup) than a color-mismatched distractor in both referentpresent and referent-absent tasks. Our data confirm that toddlers routinely access color information when trying to match spoken words with copresent visual objects, at least for concepts high in color diagnosticity. However, even for concepts high in color diagnosticity, semantic knowledge influenced toddlers' eye gaze well before color knowledge: They looked earlier at an image of cookies than at the yellow cup when they had heard "banana." This demonstrates that semantic representations are a particular central aspect of toddler's word meaning.

\section{References}

Arias-Trejo, N., \& Plunkett, K. (2009). Lexical priming effects during infancy. Philosophical Transaction of the Royal Society: B. Biological Sciences, 364, 3633-3647. doi:10.1098/rstb.2009.0146

Arias-Trejo, N., \& Plunkett, K. (2011). Lexical priming effects between 18and 24-months of age. Paper presented at the 17th Meeting of the European Society for Cognitive Psychology, San Sebastian, Spain.

Bornstein, M. H., Kessen, W., \& Weisskopf, S. (1976). Color vision and hue categorization in young infants. Journal of Experimental Psychology: Human Perception and Performance, 2, 115-129. doi:10.1037/ 0096-1523.2.1.115

Catherwood, D., Crassini, B., \& Freiberg, K. (1989). Infant response to stimuli of similar hue and dissimilar shape: Tracing the origins of the categorization of objects by hue. Child Development, 60, 752-762. doi:0009-3920/89/6003-00

Franklin, A., \& Davies, I. R. L. (2004). New evidence for infant colour categories. British Journal of Developmental Psychology, 22, 349-377. doi:10.1348/0261510041552738

Ganea, P. A. (2005). Contextual factors affect absent reference comprehension in 14-month-olds. Child Development, 76, 989-998. doi:00093920/2005/7605-0004

Graham, S. A., \& Poulin-Dubois, D. (1999). Infants' reliance on shape to generalize novel labels to animate and inanimate objects. Journal of Child Language, 26, 295-320. doi:10.1017/S0305000999003815

Grison, S., Paul, M. A., Kessler, K., \& Tipper, S. P. (2005). Inhibition of object identity in IOR: Implications for encoding and retrieving inhibition. Psychonomic Bulletin \& Review, 12, 553-558. doi:10.3758/ BF03193804

Hayne, H., Rovee-Collier, C., \& Perris, E. E. (1987). Categorization and memory retrieval by three-month-olds. Child Development, 58, 750767. doi: $10.2307 / 1130212$

Huettig, F., \& Altmann, G. T. M. (2005). Word meaning and the control of eye fixation: Semantic competitor effects and the visual world paradigm. Cognition, 96, B23-B32. doi:10.1016/j.cognition.2004.10.003

Huettig, F., \& Altmann, G. T. M. (2011). Looking at anything that is green when hearing "frog": How object surface color and stored object color knowledge influence language-mediated overt attention. The Quarterly Journal of Experimental Psychology, 64, 122-145. doi:10.1080/ 17470218.2010.481474

Huettig, F., Olivers, C. N. L., \& Hartsuiker, R. J. (2011). Looking, language, and memory: Bridging research from the visual world and visual search paradigms. Acta Psychologica, 137, 138-150. doi: 10.1016/j.actpsy.2010.07.013

Johnson, E. K., \& Huettig, F. (2011). Eye movements during languagemediated visual search reveal a strong link between overt visual attention and lexical processing in 36-months-olds. Psychological Research, 75, 35-42. doi:10.1007/s00426-010-0285-4

Johnson, E. K., McQueen, J. M., \& Huettig, F. (2011). Toddlers' languagemediated visual search: They need not have the words for it. The Quarterly Journal of Experimental Psychology, 64, 1672-1682. doi: 10.1080/17470218.2011.594165

Landau, B., Smith, L. B., \& Jones, S. S. (1988). The importance of shape in early lexical learning. Cognitive Development, 3, 299-321. doi: 10.1016/0885-2014(88)90014-7

Liu, J., Golinkoff, R. M., \& Sak, K. (2001). One cow does not an animal make: Young children can extend novel words at the superordinate level. Child Development, 72, 1674-1694. doi:10.1111/1467-8624.00372

Mani, N., \& Plunkett, K. (2007). Phonological specificity of consonants and vowels in early lexical representations. Journal of Memory and Language, 57, 252-272. doi:10.1016/j.jml.2007.03.005 
Mani, N., \& Plunkett, K. (2008). Fourteen-month-olds pay attention to vowels in novel words. Developmental Science, 11, 53-59. doi:10.1111/ j.1467-7687.2007.00645.x

Mani, N., \& Plunkett, K. (2010). In the infant's mind's ear: Evidence for implicit naming in infancy. Psychological Science, 21, 908-913. doi: 10.1177/0956797610373371

Meints, K., Plunkett, K., \& Harris, P. L. (1999). When does an ostrich become a bird? The role of typicality in early word comprehension. Developmental Psychology, 35, 1072-1078. doi:10.1037/00121649.35.4.1072

Moss, H. E., McCormick, S. F., \& Tyler, L. K. (1997). The time-course of activation of semantic information during spoken word recognition. Language and Cognitive Processes, 12, 695-732. doi:10.1080/ 016909697386664

Saylor, M. M. (2004). Twelve- and 16-month-old infants recognize properties of mentioned absent things. Developmental Science, 7, 599-611. doi:10.1111/j.1467-7687.2004.00383.x

Styles, S., \& Plunkett, K. (2011). Early links in the early lexicon: Semantically related word-pairs prime picture looking in the second year. In G.
Gaskell \& P. Zwitzerlood (Eds.), Lexical representation: A multidisciplinary approach (pp. 51-88). Berlin, Germany: Mouton de Gruyter. doi:10.1515/9783110224931.51

Swingley, D., \& Fernald, A. (2002). Recognition of words referring to present and absent objects by 24-month-olds. Journal of Memory and Language, 46, 39-56.

Wilcox, T. (1999). Object individuation: Infants'use of shape, size, pattern, and color. Cognition, 72, 125-166. doi:10.1016/S0010-0277(99)00035-9

Wilcox, T., Woods, R., Chapa, C., \& McCurry, S. (2007). Multisensory exploration and object individuation in infants. Developmental Psychology, 43, 479-495. doi:10.1037/0012-1649.43.2.479

Yee, E., \& Sedivy, J. C. (2006). Eye movements to pictures reveal transient semantic activation during spoken word recognition. Journal of Experimental Psychology: Learning, Memory, and Cognition, 32, 1-14. doi: 10.1037/0278-7393.32.1.1

Received October 25, 2011 Revision received May 9, 2012 Accepted May 11, 2012

\section{E-Mail Notification of Your Latest Issue Online!}

Would you like to know when the next issue of your favorite APA journal will be available online? This service is now available to you. Sign up at http://notify.apa.org/ and you will be notified by e-mail when issues of interest to you become available! 